PROCEEDINGS OF THE

AMERICAN MATHEMATICAL SOCIETY

Volume 120, Number 4, April 1994

\title{
THE STRUCTURE OF JOHNS RINGS
}

\author{
CARL FAITH AND PERE MENAL
}

(Communicated by Lance W. Small)

\begin{abstract}
In this paper we continue our study of right Johns rings, that is, right Noetherian rings in which every right ideal is an annihilator. Specifically we study strongly right Johns rings, or rings such that every $n \times n$ matrix ring $R_{n}$ is right Johns. The main theorem (Theorem 1.1) characterizes them as the left FP-injective right Noetherian rings, a result that shows that not all Johns rings are strong. (This first was observed by Rutter for Artinian Johns rings; see Theorem 1.2.) Another characterization is that all finitely generated right $R$-modules are Noetherian and torsionless, that is, embedded in a product of copies of $R$. A corollary to this is that a strongly right Johns ring $R$ is preserved by any group ring $R G$ of a finite group (Theorem 2.1). A strongly right Johns ring is right $F P F$ (Theorem 4.2).
\end{abstract}

\section{INTRODUCTION}

Rutter's theorem $[\mathrm{R}]$ characterizes the quasi-Frobenius $(=Q F)$ rings as right Artinian strongly right Johns. This raises the question: Are all strongly right Johns rings $Q F$ ? We do not know, but we show that the only non-Artinian right Johns rings ever constructed (in [F-M]) are not strongly right Johns. Thus, it would appear that a counterexample to the conjecture that all strongly right Johns rings are $Q F$ would have to be of larger complexity than the [F-M] examples, which interalia required Cohn's and Resco's Theorems on existentially closed skew fields and $V$-domains. A number of conditions necessary and sufficient for a right Johns ring to be Artinian, hence for a strongly right Johns ring to be $Q F$, were collected in [F-M]: $R$ semilocal; or $R$ has finite left Goldie dimension. We include these in Corollary 1.3 and add one: $J=\operatorname{rad} R$ is finitely annihilated.

We now relate strong Johns rings to class rings that are also $Q F$ when they are Artinian: A ring $R$ is right $(F) P F$ if all (finitely generated) faithful right

Received by the editors September 18, 1992.

1991 Mathematics Subject Classification. Primary 16P40, 16D70; Secondary 16L60, 16D50, $16 \mathrm{D} 40$.

The research of the first author was partially supported by three grants: The Spanish Ministry of Education and Science, the Centre Recerca Matematica, and a Rutgers University Academic Study Program (FASP) grant. The research of the second author was supported by grant CICYT PB86-0353-C02-01. This research was conducted in part during Algebra semester at Centre Recerca Matematica, Fall 1989, at Universitat Autónoma de Barcelona (UAB).

The second author is deceased (April 4, 1991; see the notes in [F-M]). 
$R$-modules are generators in the category of all right $R$-modules; equivalently, there exists an epimorphism $M^{n} \rightarrow R$, for some $n>0$, hence an isomorphism $M^{n} \approx R \oplus X$ in mod- $R$. Right $P F$ rings are rings $R$ that are injective cogenerators in the category mod- $R$ and have been characterized as the semilocal rings right self-injective rings with essential right socles. The Artinian $F P F$ rings are the $Q F$ rings. In fact, as alluded to above, Artinian $F P F$ rings are $Q F$ [F8, F9], and strongly right Johns rings are right $F P F$ (Theorem 4.2).

\section{REMARKS ON RIGHT ANNULAR RINGS AND A THEOREM OF RUTTER}

A ring $R$ is right (finitely) annular if every (finitely generated) right ideal is a right annihilator (= right annulet). A classical theorem of ring theory states that a right Noetherian ring $R$ is 2 -sided annular iff $R$ is quasi-Frobenuis $(=Q F)$. (See, e.g., [F, Chapter 24].) This shows that 2-sided Johns rings are $Q F$. Nevertheless, there exist right annular rings $R$ with just three right ideals, namely, $R, J=\operatorname{rad} R$, and 0 , which are not left Noetherian, hence not $Q F$.

We list properties of right annular rings that are either obvious or easy to prove.

(RA 1) $R$ is right annular iff every cyclic right module is torsionless. See [F4] for this and the next.

(RA 2) Every matrix ring over $R$ is right annular iff every finitely generated module is torsionless $(=R$ is right $F G T)$.

(RA 3) A left $\aleph_{0}$-injective ring is finitely right annular. (RA 3) is a theorem of Ikeda-Nakayama. (See, e.g., [F2, p. 189, 23.11].)

(RA 4) A ring $R$ is left FP-injective iff every finitely presented right $R$-module is torsionless. ((RA 4) is a theorem of Stenstrom and Jain. See [Ja].)

A ring $R$ is right $F G(T) F$ if every finitely generated (torsionless) module embeds in a free module. A right annulet $I$ is said to be finitely annihilated $(=F A)$ if $I=r(X)$ is the right annihilator of a finite subset $X$ of $R$. A necessary and sufficient condition that $I=r(X)$ where $X$ has $n$ elements is that $R / I$ embeds in $R^{n}$. Moreover, a sufficient condition for every right annulet to be $F A$ is that $R$ satisfies the acc on left annulets [F10].

(RA 5) A ring $R$ is right $F G T F$ iff every right annulet in every matrix ring $R_{n}$ is $F A$. (See, e.g., [F4] for (RA 5).)

Similarly,

(RA 6) A ring $R$ is right $F G F$ iff every matrix ring $R_{n}$ is right annular and every right ideal is $F A$.

We call a ring with the latter property right FA-annular. Thus (RA 6) states that $R_{n}$ is right $F A$-annular for all $n \leq 1$ iff $R$ is right $F G F$.

We restate (RA 6) as:

(RA 7) The f.a.e:

(RA 7a) $R$ is right $F G F$,

(RA 7b) $R_{n}$ is right $F A$-annular for all $n \geq 1$,

(RA 7c) $R$ is right $F G T F$ and $R_{n}$ is right annular for all $n \geq 1$.

It is known that every $Q F$ ring $R$ has the characterizing property: (right $G F$ ) every right module embeds in a free module.

Furthermore, by the symmetric properties of $Q F$ rings, we have: 
(RA 8) Every $Q F$ ring $R$ is right and left $G F$.

By results stated in [F3]:

(RA 9) $R$ is $Q F$ iff left and right $F G F$.

also:

(RA 10) Let $R$ be right $F G F$ ring. Then f.a.e.c.'s

(FGF 1) $R$ is $Q F$,

(FGF 2) $R$ is right Noetherian,

(FGF 3) $R$ is semilocal with essential right socle,

(FGF 4) $R$ has finite essential right socle,

(FGF 5) $R$ is right self-injective,

(FGF 6) $R$ is left $F G F$,

(FGF 7) $R$ is right and left $F A$-annular.

For a proof, see [F3]. The proof of (FGF 2) in [F3] uses John's lemma.

1.1. Theorem. For a ring $R$, the f.a.e.:

(1) $R$ is strongly right Johns.

(2) $R$ is left FP-injective and right Noetherian.

(3) $R$ is right FGT and right Noetherian.

(4) Every finitely generated right $R$-module is Noetherian torsionless.

Proof. (1) $\Leftrightarrow(3),(2) \Leftrightarrow(4)$, and (3) $\Leftrightarrow(4)$ are obvious by (RA4) and (RA2), for right Noetherian $R$.

We now prove what is essentially:

1.2. Rutter's Theorem. For a right Artinian ring, the f.a.e:

(1) $R$ is $Q F$.

(2) $R_{n}$ is ring annular for $n>1$.

(3) $R$ is strong right Johns.

Proof. (2) $\Leftrightarrow(3)$ is trivial since $R_{n}$ is right Artinian, hence right Noetherian, and $Q F$ is a Morita invariant property, i.e., is inherited by $R_{n}$.

$(2) \Rightarrow(1)$. We show this follows from (RA 10). Since $R_{n}$ is right Artinian, it satisfies

$$
(\perp \text { acc })=\text { the acc on left annulets. }
$$

But by a theorem of [F10], $\perp$ acc implies that $R_{n}$ is right $F A$. Thus, by (RA 7), $R$ is right $F G F$, hence $Q F$ by (FG 2) of (RA 10). Conversely, $(1) \Rightarrow(2)$ is obvious, since (1) implies that $R_{n}$ is $Q F$ and hence annular by (RA 10).

1.3. Corollary. Let $R$ be strongly right Johns. The fa.e.:

(1) $R$ is $Q F$.

(2) $R$ is semilocal.

(3) $R$ has finite left Goldie dimension.

(4) $R$ is left Noetherian.

(5) $R_{n}$ is right $F A$ for all $n$.

(6) $J=\operatorname{rad} R$ is right $F A$, i.e., $J=r(X)$, for a finite set $X$.

Proof. By Rutter's theorem, Theorem 1.2, to prove $Q F$ it suffices to prove $R$ is right Artinian. That (4) $\Rightarrow(3) \Rightarrow(2) \Rightarrow R$ right Artinian holds in right Johns rings (see $[\mathrm{F}-\mathrm{M}])$; and obviously $(1) \Rightarrow(4)$, and $(4) \Rightarrow(5) \Rightarrow(6)$. Also, 
(5) $\Rightarrow R$ is right $F G F$ by (RA 7), and then $R$ is $Q F$ by (RA 10), especially (FGF 2). Finally, (6) implies that $R / J$ embeds in $R^{n}$ where $n=|X|$. Now $R$ has finite essential socle by John's results in [J], so $R / J$ has the same, but being semiprime, this implies that $\mathrm{R} / \mathrm{J}$ is semisimple, so (2) holds.

\section{FINITE GROUP RINGS OVER JOHNS RINGS}

Let $G$ be a finite group. We raise the question: if $R$ is right Johns, is the group ring $R G$ ?

Using Theorem 1.1, we show the answer is affirmative assuming that $R$ is strong right Johns.

2.1. Theorem. If $R$ is a strong right John ring, then so is the group ring $R G$ of a finite group $G$.

Proof. First, $R$ right Noetherian implies $R G$ right Noetherian. Because of the natural isomorphism of functors

$$
\operatorname{Hom}_{R G}(, R G) \approx \operatorname{Hom}_{R}(, R),
$$

it is easy to see that $R$ left FP-injective implies that $R G$ is left FP-injective when $R$ is right Noetherian, so $R G$ is strong right John by Theorem 1.1.

As corollary to the proof we have:

2.2. Corollary. Let $G$ be a finite group. If $R$ is right $F G T$, then $R G$ is right FGT.

Proof. This follows from the natural isomorphism (*). To wit, if $M$ is a finitely generated $R G$-module, then $M$ is a finitely generated canonical $R$ module, and by $(*)$, the canonical map

$$
M \rightarrow R^{\mathrm{Hom}} R G^{(M, R G)}
$$

is an embedding iff the same is true of the canonical map

$$
M \rightarrow R^{\mathrm{Hom}} R^{(M, R)} .
$$

Thus, $R$ right $F G T$ implies that $R G$ is right $F G T$.

\section{STRUCTURE OF STRONGLY JOHNS RINGS}

A module $M$ is finitely embedded (= f.e.) provided $M$ has finite essential socle. It is known that $M$ is f.e. iff $M$ has the finite intersection property (= f.i.p.), namely, if $\left\{S_{i}\right\}_{i \in I}$ is any family of submodules and if $\bigcap_{i \in I} S_{i}=0$, then there is a finite subset $A \subseteq I$ with $\bigcap_{a \in A} S_{a}=0$. (See [F2, p. 69] for background and references.) We need the following elementary property of f.e. modules.

3.1.A. Proposition. If $M$ is a f.e. right $R$-module and if $M$ is contained in a product $N=\prod_{i \in I} N_{i}$ of right $R$-modules, then there is a finite subset $A \subseteq I$ such that $M \hookrightarrow \bigoplus_{a \in A} N_{a}$.

Proof. Let $\rho_{i}: N \rightarrow N_{i}$ be the canonical projections from the direct product, and let $\bar{\rho}_{i}: M \rightarrow N_{i}$ be the induced map for all $i \in I$. Since $\bigcap_{i \in i} \operatorname{ker} \rho_{i}=0$, and since $M$ is f.e., for some finite subset $A$ of $I$ we have $\bigcap_{a \in A}$ ker $\bar{\rho}_{a}=0$, and then the direct sum of the maps $\left\{\bar{\rho}_{a}\right\}_{a \in A}$ is an embedding $M \hookrightarrow \bigoplus_{a \in A} N_{a}$. 
3.1.B. Corollary. Every finitely embedded torsionless right $R$-module $M$ embeds in free module $R^{n}$ of finite rank $n$.

Proof. Since $M$ is torsionless and then embeds in a direct product of copies of $R$, the corollary follows from Proposition 3.1.A.

3.2. Theorem. If $R$ is strongly right Johns, then the injective hull $E=E\left(R_{R}\right)$ of $R$ is a flat right $R$-module.

Proof. The proof requires a theorem of Rutter $[R]$ to the effect that an injective module is flat iff every finitely generated submodule embeds in a flat module. Actually we can prove more: every finitely generated submodule $M$ of $E$ embeds in a free $R$-module. For $R$ is finitely embedded [J], hence so is $E$ whence $M$. By Theorem 1.1, each finitely generated right $R$-module is torsionless, hence by Corollary 3.1.B, $M$ embeds in $R^{n}$. This proves what was needed.

3.3. Lemma. If $A$ is a semiprime right Goldie ring with right quotient ring $Q=Q_{\mathrm{cl}}^{r}(A)$, then any torsionfree divisible right A-module $F$ is injective and a canonical $Q$-module.

Proof. Let $G=E\left(F_{A}\right)$. Then $G$ is also torsionfree, for if $0 \neq g \in G$ and if $a \in A$ is such that $g a=0$, we can find a regular element $d \in A$ such that $0 \neq g d \in F$. (This follows because the conductor $(g: F)$ of $g$ to $F$ is an essential right ideal and every essential right ideal in a semiprime right Goldie ring contains a regular element.)

Next, since $F$ is torsionfree over $A, F$ is a canonical $Q$-module, so we may apply $d^{-1} a$ to $g d$ to get

$$
(g d)\left(d^{-1} a\right)=g a=0 .
$$

But $F$ is torsionfree, contradicting $g d \neq 0$. Thus, $d^{-1} a=0$, so $a=0$, hence $G$ is torsionfree. But, since $F$ is divisible, $g d=x d$ for $x \in F$, so

$$
(g-x) d=0
$$

whence $g-x=0$, that is, $g=x \in F$. Therefore, $F=G$ is injective.

We shall need the next proposition which was originally in [F-M] but excised in keeping with instructions from the referee and the corresponding editor.

3.4. Proposition. Let $R$ be right Johns, and let $\bar{R}=R / J$, where $J$ is the Jacobson radical of $R$. Then:

(i) If $a \in R$, then $r_{R}(a)=0$ if and only if $r_{R}(\bar{a})=0$.

(ii) If $\bar{R}$ is a domain, then $S=\operatorname{soc} R$ is a minimal right ideal of $R$ and the unique simple right $R$ module.

Proof. (1) Assume $r_{R}(a)=0$, and let $a b \in J$ for some $b \in R$. Then, by Lemma 2.2 of $[\mathrm{F}-\mathrm{M}], a(b \cdot \operatorname{soc} R)=0$. Hence $b \cdot \operatorname{soc} R=0$, so $b \in J=$ $l(\operatorname{soc} R)$ by Lemma 2.2 of [F-M] again. Thus, $r_{R}(\bar{a})=0$.

Conversely, let $a \in R$ be such that $r_{R}(\bar{a})=0$. Then, since $\bar{R}$ is semiprime right Noetherian, necessarily $l_{R}(\bar{a})=0$, hence

$$
J=\{x \in R \mid x a \in J\}=(x: J) .
$$

Since $J=l_{R}(\operatorname{soc} R)$, it follows that $J=l_{R}(a \cdot \operatorname{soc} R)$, so we see that $\operatorname{soc} R=a \cdot \operatorname{soc} R$ since every right ideal of $R$ is a right annihilator. 
Since $a$ therefore induces an epimorphism of the Noetherian module $\operatorname{soc} R$, $r_{R}(a) \cap(\operatorname{soc} R)=0$. Since $\operatorname{soc} R$ is an essential right ideal of $R$ by a lemma of Johns [J, Lemma 2], $r_{R}(a)=0$, proving (i).

(ii) Let $V$ be a right ideal of $R$ properly contained in $S$. Then, by Lemma 2 of [J], $l_{R}(V) \supset l_{R}(S)=J$. By (i) above, any $a \in l_{R}(V) \backslash J$ satisfies $r_{R}(a)=0$, a contradiction unless $V=0$. Thus, $S$ is a minimal right ideal. Since every right ideal of $R$ is a right annihilator, every simple right $R$-module $W$ of $R$ embeds in $R$, hence coincides with $S$ since $S$ is essential. Thus, $S$ is the unique simple $R$-module.

3.5. Theorem. If $R$ is right Johns, $S=\operatorname{soc} R_{R}, Y=\operatorname{ann}_{E} S$, and $\bar{E}=E / Y$, then $\bar{E}$ is an injective right A-module.

Proof. Since $J$ is the left annihilator in $R$ of $S=\operatorname{soc} R_{R}$ (see proof of Proposition 3.4), $Y \supseteq E J$, so $Y$ is a canonical $A$-module. Now $E$ (and any injective module) is divisible by every right regular element $a$ of $R$. By (i) of Proposition 3.4, every (right) regular element $\bar{a}$ of $A=\bar{R}$ lift to a right regular element $a \in R$. Thus, $E$ is divisible (by regular elements of $A$ ). Since $|S|<\infty$ and $a S \approx S$, it follows that $a S=S$ for any $a \in R$ above.

Now if $\bar{x} \bar{a}=0$ for $\bar{x} \in \bar{E}$ and regular $\bar{a} \in A$, then $x a \in Y$, hence

$$
0=(x a) S=x(a S)=x S,
$$

so $x \in Y$, i.e., $\bar{x}=0$. This proves that $\bar{E}$ is torsionfree divisible over $A$, hence that $E$ is injective by Lemma 3.3.

3.6. Theorem. If $R$ is strongly right Johns and $A=\bar{R}=R / J$ is a domain, then

$$
Q=Q_{\mathrm{cl}}^{r}(A)=Q_{\mathrm{cl}}^{\ell}(A) \stackrel{\text { can }}{\approx} \text { End } V_{R}=\text { End } V_{A}
$$

where $V$ is the unique simple $R$-module.

Proof. Since $R$ is right Johns and $A=R / J$ is a domain, $V=\operatorname{soc} R$ is simple and the unique simple right $R$-module by Proposition 3.4. By Schur's lemma, $K=$ End $V_{R}=$ End $V_{A}$ is a field. If $\operatorname{dim}_{K} V=1$, then $A=K$ and the result is trivial. Otherwise, we may suppose for every $0 \neq v \in V$ that $K v \cap K d v=0$ and hence that

$$
A v \cap A d v=0 .
$$

Then, by a property of left $\aleph_{0}$-injective rings (which follows since $R$ is left FP-injective by Theorem 1.1)

$$
r_{R}(v)=r_{R}(v)+r_{R}(d v)=R
$$

(see, e.g., the theorem of Ikeda-Nakayama in [F2, p. 139]). Thus, $v=0$, a contradiction. This similarly implies that, for every $0 \neq v \in V$,

$$
A d_{0} v \cap A b v \neq 0 .
$$

Write $a d_{0} v=b v$ for some $a \in A$. Then $a d_{0}=b$ (since ${ }_{K} V$ is a vector space), hence

$$
d_{0}=a^{-1} b \in Q_{c \ell}^{r}(A),
$$

i.e., $K=Q_{\mathrm{cl}}^{r}(A)$. Using the same argument with $d_{0}=c d$, for $0 \neq c, d \in A$, we get $a^{-1} b=c d^{-1}$, hence that

$$
K=Q_{\mathrm{cl}}^{\ell}(A)=Q_{\mathrm{cl}}^{r}(A) .
$$


For a bimodule $V$ over a ring $A$, we let $(A, V)$ denote the trivial, or splitnull extension; namely, the ring consisting of all matrices $\left(\begin{array}{ll}a & \nabla \\ 0 & a\end{array}\right)$ with $a \in$ $A, \nabla \in V$, and the usual matrix multiplication.

3.7. Remark. The right Johns ring $R=(A, V)$ constructed in [F-M] is not strongly right Johns.

For in this example, $A=D \otimes_{C} C(X)$, for an existentially closed field $D$ with center $C$, and $V=D$ is an $A$-bimodule, so that $D \approx$ End $V_{A}$ has center $C$, whereas the center of $Q_{\mathrm{cl}}(A)=C(X)$. Thus, since $C$ is algebraically closed, then $C \not \approx C(X)$, hence $Q_{\mathrm{cl}}(A) \neq$ End $V_{A}$ as required by Theorem 3.6 in strongly right Johns ring.

3.8. Theorem. Let $R$ be strongly right Johns, $A=R / J$, where $J=\operatorname{rad} R$, and $S=\operatorname{soc} R$. Then for $E=E\left(R_{R}\right)$, we have:

(i) $Y=\operatorname{ann}_{E} S=E J$,

and $\bar{E}=E / E J$ is torsionfree over $A$, and an injective right $Q$-module, where $Q=Q_{\mathrm{cl}}^{r}(A)$. Moreover,

(ii) $Q_{A}$ is flat, and

(iii) $Q=Q_{\mathrm{cl}}^{\ell}(A)$.

Proof. Since $A$ is semiprime right Noetherian (hence Goldie), then $Q=Q_{\mathrm{cl}}^{r}(A)$ exists by, e.g., [F1, Chapter 9] and is semisimple (theorem of A. W. Goldie). Moreover, any torsionfree divisible right module is canonically a right $Q$ module (see Lemma 3.3).

Let $y \in Y=\operatorname{ann}_{E} S$. By Corollary 3.1.B,

$$
y R \approx R / \operatorname{ann}_{R} y
$$

embeds in a free right $R$-module of finite rank, hence

$$
\operatorname{ann}_{R} y=r_{R}(X)
$$

is the right annihilator in $R$ of a finite subset $X$ of $R$. By the double annihilator condition (d.a.c.) for (quasi) injective modules (e.g., [F2, Chapter 19, Theorem 19.10, p. 66])

$$
\operatorname{ann}_{E} \operatorname{ann}_{R} M=M
$$

for any finitely generated left submodule of the left module $E$ over $\Lambda=$ End $E_{R}$. Since

$$
\operatorname{ann}_{R} \Lambda X=\operatorname{ann}_{R} y=\operatorname{ann}_{R} \Lambda y,
$$

it follows that $y \in \Lambda X$. But,

$$
r_{R}(X)=\operatorname{ann}_{R} y \supseteq \operatorname{ann}_{R} Y \supseteq S,
$$

hence,

$$
X \subseteq l_{R} r_{R}(X) \subseteq l_{R}(S)=J .
$$

(The right equality is [F-M, Lemma 2.2].) So,

$$
y \in \Lambda X \subseteq \Lambda(1) J=E J,
$$

proving (i).

(ii) and (iii). By a theorem of Levy [L] and Goodearl [G], (ii) $\Rightarrow$ (iii), so we proceed to prove (ii). By Theorem 3.2, $E_{R}$ is flat, and hence $E / E I$ is a flat $(R / I)$-module for any ideal $I$ of $R$, so $E / E J$ is a flat $A$-module. 
Since $\bar{E}=E / E J$ is a torsionfree divisible, in fact, injective, right $A$-module by Theorem 3.5 , and canonically a module over $Q$, it follows that the least generator $Q_{0}$ of mod- $Q$ is a direct summand of $\bar{E}$, hence $\left(Q_{0}\right)_{A}$ whence $Q_{A}$ is flat.

3.9. Corollary. If $R$ is strongly right Johns, then $S=\operatorname{soc} R_{R}$ is an injective right and left $A$-module, where $A=R / J$.

Proof. By Theorem 2.3 of [F-M], $A$ is a right $V$-ring, i.e., every simple right $A$ module is injective. Since $R$, whence $A$, is right Noetherian, every semisimple right $A$-module is injective, thus $S_{A}$ is injective. By Lemma 3.3 if $\bar{a} \in A=$ $R / J$, where $a \in R$, then $r_{R}(\bar{a})=0$ implies $r_{R}(a)=0$, hence ${ }_{A} S$ is torsionfree. Also, the fact that $S_{A}$ has finite length and that $S \approx a S$ implies that $a S=S$, so ${ }_{A} S$ is divisible. By Theorem 3.8 (iii), $A$ is left Ore, hence left Goldie, so by Proposition 3.4, then ${ }_{A} S$ is injective and canonically an injective left $Q$-module, where $Q=Q_{\mathrm{cl}}^{r}(A)=Q_{\mathrm{cl}}^{\ell}(A)$.

3.10. Theorem. Let $R$ be strongly right Johns, with $J^{2}=0, S=\operatorname{soc} R_{R}$, and suppose that

$$
Q \stackrel{\text { can }}{\approx} \text { End } S_{R}
$$

(e.g., if $A=R / J$ is a domain: see Theorem 3.6). Then, $E J=J$ and $E / J \approx$ $Q$.

Proof. By Theorem 3.8, $\bar{E}=E / E J$ contains a copy of $Q$. For any ideal $I, a_{E} I$ is an injective $(R / I)$-module; in particular,

$$
F_{1}=\operatorname{ann}_{E} J
$$

is an injective $A$-module. Since $E$ is an essential extension of $S_{R}$, then $F_{1}$ is essential over $S_{A}$, hence $F_{1}=S$ by injectivity of $S$ (see Corollary 3.9).

Let $R \subseteq E_{1} \subseteq E$ be such that

$$
E_{1} / J \approx Q
$$

Now $E_{1}$ exists since

$$
S=J \subseteq E J \subseteq \operatorname{ann}_{E} J=S
$$

(note $S=J$ by loc. cit.). Thus,

$$
E J=J \text { and } \bar{E}=E / J,
$$

so (1) exists since $\bar{E}$ contains a copy of $Q$.

Let $y \in E$. Then, by (2)

$$
y S \subseteq S=\operatorname{ann}_{E} J .
$$

So $y$ induces $\bar{y} \in K=$ End $S_{R}=$ End $S_{A}$. But $Q \subseteq K$ canonically, and by the assumption $Q=K$, we obtain $q \in Q \subseteq E$ such that $\bar{y}=\bar{q}$, i.e.,

$$
s=y-q \in \operatorname{ann}_{E} S=\operatorname{ann}_{E} J=S,
$$

so $y=q+s \in E_{1}$. This proves that $E=E_{1}$, hence $\bar{E} \approx Q$.

3.11. Corollary. If $R$ in the theorem is the split-null extension $R=(A, W)$ (as in $[\mathrm{F}-\mathrm{M}])$, where $A=R / J$ and $W=\operatorname{Soc} R_{R}$, then:

$$
E=(Q, W) \text {. }
$$


Moreover, in this case

End $E_{R} \approx\left(Q, \operatorname{Hom}\left(Q_{A}, W_{A}\right)\right)$.

((1) and (2) are trivial extensions.)

Proof. (1) Straightforward application of Theorem 3.10. (2) follows by an easy calculation.

\section{Strongly Johns Rings ARE FPF}

A ring $R$ is right $(F) P F$ if every (finitely generated) faithful right $R$-module $M$ is a generator of mod- $R$, the category of all right $R$-modules. This happens iff there is a (finite) direct sum of copies $M$ and an epimorphism $M^{n} \longrightarrow R$, hence an isomorphism

$$
M^{n} \approx R \oplus X \quad(\text { in } \bmod -R) .
$$

The relation between $F P F, P F$, and $Q F$ rings is studied in [F5-F9].

4.1. Proposition. If $J=\operatorname{rad} R$ is nilpotent and $M$ is a faithful torsionless right $R$-module, then the trace ideal $T(M) \nsubseteq J$.

Proof. Suppose $J^{n}=0$ and $J^{n-1} \neq 0$. Since $M$ is faithful, $M J^{n-1} \neq 0$, and since $M$ is torsionless, there exist $f \in M^{*}=\operatorname{Hom}_{R}(M, R)$ such that

$$
f\left(M J^{n-1}\right) \neq 0 \text {. }
$$

Then

$$
T(M) J^{n-1}=T\left(M J^{n-1}\right)=\sum_{f \in M^{*}} f\left(M J^{n-1}\right) \neq 0,
$$

proving that $T(M) \nsubseteq J$.

\subsection{Theorem. A strongly right Johns ring $R$ is right FPF.}

Proof. By Theorem 1.1(4) and [F-M, Lemma 2.2], if $M$ is any finitely generated faithful right $R$-module, then $T=T(M) \nsubseteq J$ by Proposition 4.1. Since $A=$ $R / J$ is a right Noetherian right $V$-ring, by [F-M], then $A$ is a finite product of simple rings. (See [F1, Chapter 7, Theorem 7.36A, p. 357].) Suppose $T P \subseteq J$ for an ideal $P$ of $R$ containing $J$. Now $J=l_{R}(S)$, where $S=\operatorname{soc} R_{R}$ by a result of John stated in $[\mathrm{F}-\mathrm{M}]$, so $T P S=0$, and then, as in the proof of Proposition 4.1, $P S=0$, so $P \subseteq J=l(S)$, whence $P=J$. This proves that $T=T / J$ is a faithful module over $A$. Since $A$ is finite a product of simple ideals, $\bar{T}$ must be a finite product of a collection of these, so $\bar{T}$ is faithful only if $\bar{T}=A$. This proves that $T=R$, so $R$ is right $F P F$.

4.3. Corollary. If, in Theorem 4.2, $A=R / J$ is a (right $\&$ left) $P I D$ and $J^{2}=0$, then every finitely generated right $R$-module $M$ is (up to isomorphism) a unique direct sum of indecomposable cyclic modules. In fact, there exist unique nonnegative integers $m$ and $n$ such that

$$
M \approx R^{(n)} \oplus A^{(m)} \oplus M_{0}
$$

where $M_{0}$ is a semisimple (hence injective) module of finite length. Moreover, every projective right $R$-module is free.

Proof. The proof of Proposition 4.1 shows that for every finitely generated faithful right module $M$ there exists a map $f: M \rightarrow R$ such that $f(M) \nsubseteq J$. 
Using the fact that $A$ is a PID and Proposition 3.4, it follows that $f(M)=a R$ for some $a \in R \backslash J$ and that $r_{R}(a)=0$, hence $M$ maps epically onto R. Thus, by an induction argument, for any finitely generated $R$-module $M$,

$$
M \approx R^{(n)} \oplus Y
$$

where $Y$ is unfaithful over $R$, and possibly $n=0$ or $Y=0$. In this case, since $J=\operatorname{soc} R$ is simple by (ii) of 3.4 (and its proof, using $J^{2}=0$ ), then $Y$ is an $A$-module, and by the known theory of modules over PID's

$$
Y \approx A^{(m)} \oplus M_{0}
$$

where $M_{0}$ is a torsion hence Artinian right $A$-module, and a direct sum cyclic modules. However, since $A$ is a right $V$-domain [F-M], then $M_{0}$ is actually semisimple of unique finite length. Moreover, $m$ and $n$ are unique since $A$ is a PID, and $R$ is right Noetherian hence has invariant basis number for free $R$-modules. This proves that $M$ is a unique direct sum of cyclic $R$-modules.

Finally, if $M$ is a finitely generated projective module, then $M$ is free:

$$
M \approx R^{n}
$$

since $R$ has no nontrivial idempotents. $(A=R / J$ has none, and idempotents lift.)

\section{ADDENDUM}

(Added May 1993) Using the method of proof of Proposition 4.1 one can prove

Proposition 4.1*. Let $R$ be a ring such that every ideal $I \neq R$ has nonzero annihilator $I^{\perp}$. Then every torsionless faithful right $R$-module $M$ generates $\bmod -R$.

Corollary 4.2*. If $R$ is a right cogenerator ring, then $R$ is right $P F$ iff $I^{\perp} \neq 0$ for every ideal $I \neq R$.

Proof. If $R$ is right PF, then by a theorem of Kato, every maximal left ideal $L$ has $L^{\perp} \neq 0$ (see [F8, Corollary 11]). Thus, if $I$ is an ideal $\neq R$, then $I$ is contained in a maximal left ideal $L$ and hence $I^{\perp} \supset L^{\perp} \neq 0$.

Conversely, if $R$ is a right cogenerator ring, then every right $R$-module $M$ is torsionless, hence faithful modules generate mod $-R$ by Proposition $4.1^{*}$, so $R$ is right PF.

\section{REFERENCES}

[F] C. Faith, Algebra I: Rings, modules and categories, corrected reprint 1981, Springer-Verlag, Berlin, Heidelberg, and New York, 1973.

[F2] _ Algebra II: Ring theory, Springer-Verlag, New York, 1976.

[F3] _ Embedding modules in projectives, Advances in Non-Commutative Ring Theory, Lecture Notes in Math., vol. 951, Springer-Verlag, New York, 1982, pp. 21-39.

[F4] _ Embedding torsionless modules in projectives, Pub. Soc. Math. 34 (1990), 379-387.

[F5] - Self-injective rings, Proc. Amer. Math. Soc. 77 (1979), 157-164.

[F6] - Commutative FPF rings arising as split null extensions, Proc. Amer. Math. Soc. 90 (1984), 181-185.

[F7] _ When self-injective rings are $Q F: A$ report on a problem, preprint. 
[F8] Injective cogenerator rings and a theorem of Tachikawa, Proc. Amer. Math. Soc. 60 (1976), 25-30.

[F9] _ Semiperfect Prüfer rings and FPF rings, Israel J. Math. 26 (1977), 166-177.

[F10] __ Rings with ascending condition on annihilators, Nagoya Math. J. 27 (1966), 179-191.

[F-M] C. Faith and P. Menal, A counter example to a conjecture of Johns, Proc. Amer. Math. Soc. 116 (1992), 21-26.

[G] K. R. Goodearl, Embedding non-singular modules in free modules, J. Pure Appl. Algebra 1 (1971), 275-279.

[J] B. Johns, Annihilator conditions in Noetherian rings, J. Algebra 49 (1977), 222-224.

[Ja] S. Jain, Flat and FP-injectivity, Proc. Amer. Math. Soc. 41 (1973), 437-422.

[L] L. S. Levy, Torsion-free and divisible modules over non-integral domains, Canad. J. Math. 15 (1963), 132-151.

[R] E. A. Rutter, A characterization of QF-3 rings, Pacific. J. Math. 51 (1974), 533-653.

199 Longview Drive, Princeton, New Jersey 08540 